\title{
Radiotherapy for mandibular metastases from hepatocellular carcinoma: a single institutional experience
}

\author{
Jongmoo Park', Sang Min Yoon ${ }^{2}$ \\ ${ }^{1}$ Department of Radiation Oncology, Kyungpook National University Chilgok Hospital, Daegu, Korea \\ ${ }^{2}$ Department of Radiation Oncology, Asan Medical Center, University of Ulsan College of Medicine, Seoul, Korea
}

\begin{abstract}
Purpose: A mandibular metastasis is uncommon in patients with hepatocellular carcinoma (HCC). We report the clinical features of this rare lesion and evaluate the effectiveness of radiotherapy in affected patients.

Materials and Methods: We retrospectively reviewed our institutional medical records for HCC patients who received radiotherapy for bone metastasis, and included cases of mandible metastasis. The clinical features of these cases, and the characteristics and outcomes of the treatments were assessed.

Results: A total of 1,498 patients with a bone metastasis from HCC were treated with radiotherapy between July 1998 and April 2012 at our institution. We identified 9 patients $(0.6 \%)$ in this cohort that received radiotherapy for a mandibular metastasis. The condyle was the most common location of mandibular metastasis. The median radiation dose was $40 \mathrm{~Gy}$ (range, 27.5 to $60 \mathrm{~Gy}$ ), with a daily dose of 2-3 Gy. All of these 9 patients died during a median follow-up of 9 months (range, 1 to 19 months). Symptom relief was achieved in 7 of 8 patients who completed radiotherapy. Tumor size reduction was observed in 2 of 4 patients who underwent radiologic evaluation after radiotherapy.

Conclusions: Metastasis to the mandible from HCC has a poor prognosis. Radiotherapy can be an effective local treatment option for symptomatic relief in these cases.
\end{abstract}

Keywords: Hepatocellular carcinoma, Mandible, Metastasis, Radiotherapy

\section{Introduction}

Hepatocellular carcinoma (HCC) is the sixth most common malignancy and the second most common cause of death from cancer worldwide [1]. With the advances in diagnosis and treatments for HCC, survival outcomes have considerably improved over time. However, the improvement in intrahepatic tumor control and prolonged survival has led to an increased incidence of extrahepatic metastasis [2]. Bone is one of the most frequent sites of metastasis from $\mathrm{HCC}$, followed by the lung and lymph nodes [2,3].

The typical radiologic findings for a bone metastasis from HCC are soft-tissue mass formations within the osteolytic bone changes, which may produce pain, swelling and local mass effects that hinder the patient's quality of life (0oL) [4]. Radiotherapy can be used as a palliative treatment modality in these cases and its efficacy in this regard has been reported in previous studies $[4,5]$. The most common sites of a bone

Received 20 August 2019, Revised 11 December 2019, Accepted 16 December 2019.

Correspondence: Sang Min Yoon, Department of Radiation Oncology, Asan Medical Center, University of Ulsan College of Medicine, 88, Olympic-ro 43-gil, Songpa-gu, Seoul 05505, Korea. Tel: +82-2-3010-5615, Fax: +82-2-3010-6950, E-mail: drsmyoon@amc.seoul.kr (http://orcid.org/0000-0002-5138-3051)

(c) This is an Open Access article distributed under the terms of the Creative Commons Attribution Non-Commercial License (http://creativecommons.org/ licenses/by-nc/4.0/) which permits unrestricted non-commercial use, distribution, and reproduction in any medium, provided the original work is properly cited.

www.e-roj.org 
metastasis from HCC are the spine, pelvis, rib, and long bone. There are also some other less common metastatic sites such as the mandible, which was first reported by Dick et al. [6] in 1957 and has since been described in several case reports and small case series [7-13]. Due to its rarity however, its clinical features and prognosis are not well established. Consequently, there have been few reports on the role of radiotherapy in these rare cases, which is the main palliative treatment for a bone metastasis from HCC.

We have here compiled the cumulative experiences from our institution on the clinical features of mandibular metastases from HCC and the results of radiotherapy in patients with these rare metastatic lesions.

\section{Methods and Materials}

\section{Patients}

This retrospective study was approved by the Institutional Review Board of Asan Medical Center (No. 2019-1055), and the requirement for informed consent was waived due to the retrospective nature of the analyses. We identified HCC patients with mandibular metastasis who underwent radiotherapy as a palliative treatment at our institution between July 1998 and April 2012. Patients were included in this study if they met the following criteria: (1) HCC diagnosis was made by histological confirmation or using the imaging criteria of the American Association for the Study of Liver Disease [14]; (2) mandibular metastasis confirmed by either biopsy or imaging studies such as computed tomography (CT), magnetic resonance images (MRI), positron emission tomography (PET), or bone scan; and (3) radiotherapy was received for the mandibular bone lesion.

\section{Radiotherapy}

CT simulation was performed in the supine position, with a thermoplastic mask for immobilization, using a 16 slice CT (GE LightSpeed RT 16; GE Healthcare, Waukesha, WI, USA). The gross tumor volume (GTV) was defined as a contrastenhancing metastatic tumor and osteolytic, osteoblastic or mixed bone lesions on planning CT. The clinical target volume (CTV) included the GTV with a 1 to $2 \mathrm{~cm}$ expansion in the mandible bone marrow to cover microscopic infiltrations. The planning target volume (PTV) was generated through a 0.5 $\mathrm{cm}$ expansion of the CTV in all directions. Three-dimensional conformal radiotherapy planning was done using the radiotherapy planning system (Eclipse; Varian Medical Systems, Palo Alto, CA, USA) and treatment was delivered with 6-15
MV photon beams using a linear accelerator (Varian Medical Systems). The prescribed radiation doses were determined with regard to the treatment aims and minimizing toxicity to the surrounding organs including the salivary glands, oral mucosa, brain, and spinal cord.

\section{Evaluation}

After the completion of radiotherapy, the patients were followed up regularly (at 1 month after treatment and at 2- to 3-month intervals thereafter to evaluate the disease status). Pain response was defined using international consensus endpoints to assess pain severity and analgesic consumption [15]. Pain was assessed by the worst numeric pain rating scale (NRS) for the previous 3 days. A complete response (CR) was defined as 0 points on the NRS without increases in the analgesics intake (i.e., maintenance or reduction of the dose), a partial response (PR) as a reduction of 2 or more points on the NRS, a progressive disease (PD) as an increase of 2 or more points on the NRS without reduced daily oral morphine, and stable disease (SD) as pain between the PR and PD level that persisted after treatment. In patients undergoing imaging studies such as $C T, M R I, P E T / C T$, the radiologic response was also evaluated based on the Response Evaluation Criteria in Solid Tumor (RECIST version 1.1). Pain and radiologic responses were evaluated at 1 to 6 months after the completion of radiotherapy and the best responses were recorded as each response. The follow-up duration was calculated from the start of radiotherapy to the date of death or last follow-up.

\section{Results}

Nine patients $(0.6 \%)$ with a mandibular metastasis were included in this analysis among a total of 1,498 HCC cases who underwent radiotherapy for bone metastasis during the study period. The characteristics of these 9 subjects are summarized in Table 1. The median age at the diagnosis of mandibular metastasis was 52 years (range, 49 to 64 years). All of the study patients had a chronic hepatitis B virus infection as the cause of their liver disease. The intrahepatic lesions were controlled with transarterial chemoembolization in 5 patients, whereas 3 patients had multiple viable HCCs and 1 had a single viable HCC in the liver. All patients except one had other synchronous extrahepatic metastases in the lung, adrenal gland, lymph node, or other bones. The most frequent symptom of the mandibular metastasis was pain and the most common site of this lesion was the condyle $(n=5$ $55.6 \%)$, followed by the ramus $(n=3 ; 33.3 \%)$, and angle $(n=$ 
Table 1. Patient characteristics and summary of treatments

\begin{tabular}{|c|c|}
\hline Characteristic & Value \\
\hline \multicolumn{2}{|l|}{ Sex } \\
\hline Male & $8(88.9)$ \\
\hline Female & $1(11.1)$ \\
\hline Age (yr) & $52(49-64)$ \\
\hline \multicolumn{2}{|l|}{ Viral etiology } \\
\hline Hepatitis B virus & $9(100)$ \\
\hline \multicolumn{2}{|l|}{ Status of intrahepatic control } \\
\hline Yes & $5(55.6)$ \\
\hline No & $4(44.4)$ \\
\hline \multicolumn{2}{|c|}{ Other synchronous extrahepatic metastases } \\
\hline Yes & 8 (88.9) \\
\hline No & $1(11.1)$ \\
\hline \multicolumn{2}{|l|}{ Symptoms ${ }^{a}$} \\
\hline Pain & 8 (88.9) \\
\hline Difficulty in mouth opening & $5(55.6)$ \\
\hline Swelling & $3(33.3)$ \\
\hline Numbness & $2(22.2)$ \\
\hline \multicolumn{2}{|l|}{ Site of mandibular metastasis } \\
\hline Condyle & $5(55.6)$ \\
\hline Ramus & $3(33.3)$ \\
\hline Angle & $1(11.1)$ \\
\hline \multicolumn{2}{|l|}{ Sorafenib treatment } \\
\hline Yes & $4(44.4)$ \\
\hline No & $5(55.6)$ \\
\hline Tumor size, longest diameter $(\mathrm{cm})$ & $3.5(1.2-5.0)$ \\
\hline \multicolumn{2}{|l|}{ Radiotherapy dose (Gy) } \\
\hline Total dose & $40(27.5-60)$ \\
\hline Fraction size & $2.5(2-3)$ \\
\hline EOD2 $(\alpha / \beta=10)$ & $41.7(28.7-60)$ \\
\hline
\end{tabular}

Values are presented as number of patients (\%) or median (range). EOD2, equivalent dose in 2 Gy fractions.

a) Percentages were summed to over 100\% because the patients had one or more symptoms.

$1 ; 11.1 \%$ ). The median tumor size of the mandibular metastasis was $3.5 \mathrm{~cm}$ (range, 1.2 to $5.0 \mathrm{~cm}$ ). Pathologic confirmation of a metastasis from HCC was performed in 3 patients. Fig. 1 presents representative radiologic findings of a metastatic HCC derived from the ramus of mandible in a study patient.

Study patient characteristics and treatment results are summarized in Table 2. Sorafenib was administered as a systemic treatment in 2 patients prior to radiotherapy and in 2 patients after radiotherapy who showed disease progression at other metastatic sites. The median follow-up period was
9 months (range, 1 to 19 months), with no patients having survived at the time of analysis due to HCC progression. The median radiation dose was $40 \mathrm{~Gy}$ (range, 27.5 to $60 \mathrm{~Gy}$ ), with a daily dose of 2-3 Gy. The equivalent dose in $2 \mathrm{~Gy}$ fractions (EQD2, $\alpha / \beta=10$ ) was $41.7 \mathrm{~Gy}$ (range, 28.7 to $60 \mathrm{~Gy}$ ). Eight patients completed the planned radiotherapy, but 1 patient died before the end of this treatment due to PD. Of the 8 patients who completed the radiotherapy, complete pain relief was achieved in 7 cases between 3 and 6 months after completion of radiotherapy. The radiologic response could be evaluated in 4 patients, 2 of whom showed partial response (with a total dose of $50 \mathrm{~Gy}$ and $60 \mathrm{~Gy}$ ) and 2 who had stable disease (with a total dose of 37.5 Gy and 39 Gy) during the follow-up periods.

\section{Discussion and Conclusion}

A mandibular metastasis from HCC is an extremely rare clinical condition. The mandible is an uncommon metastatic site from malignancies and accounts for less than 1\% of all oral cancers [16]. Hirshberg et al. [17] have previously conducted a literature review of oral cavity metastases in 673 cases, among which 455 had a jawbone metastasis that most frequently involved the mandible. Notably however, only 23 (5.1\%) of those cases of jawbone metastases arose from the liver. Because of its rarity, only 9 patients $(0.6 \%)$ received palliative radiotherapy for a mandibular metastasis among the 1,498 cases who received radiotherapy for bone metastasis from HCC at our institution. To our knowledge, only 77 cases have been described in the literature since a mandibular metastasis from HCC was first reported in 1957 by Dick et al. [6] (Supplementary Table S1).

As noted by Junquera et al. [8], a mandibular metastasis is known to spread through two different pathways. The first of these involves a metastatic dissemination that develops in the lung through the portal vein and subsequently spreads to the mandible region. Hence, a mandibular metastasis through this pathway usually represents late stage cancer. The prevalence of this could be underestimated because of patient deaths from an advanced stage HCC before a diagnosis of mandibular metastasis could be made. The other pathway involves communication between the portal and cerebrospinal venous system via the vertebral venous system, known as the Batson's venous plexus as the bypass of valve-bearing systemic venous system $[8,18]$. This route enables a bypass of the lung filtration and a direct spread of the metastatic cancer cells in the cranial direction without pulmonary involvement. 

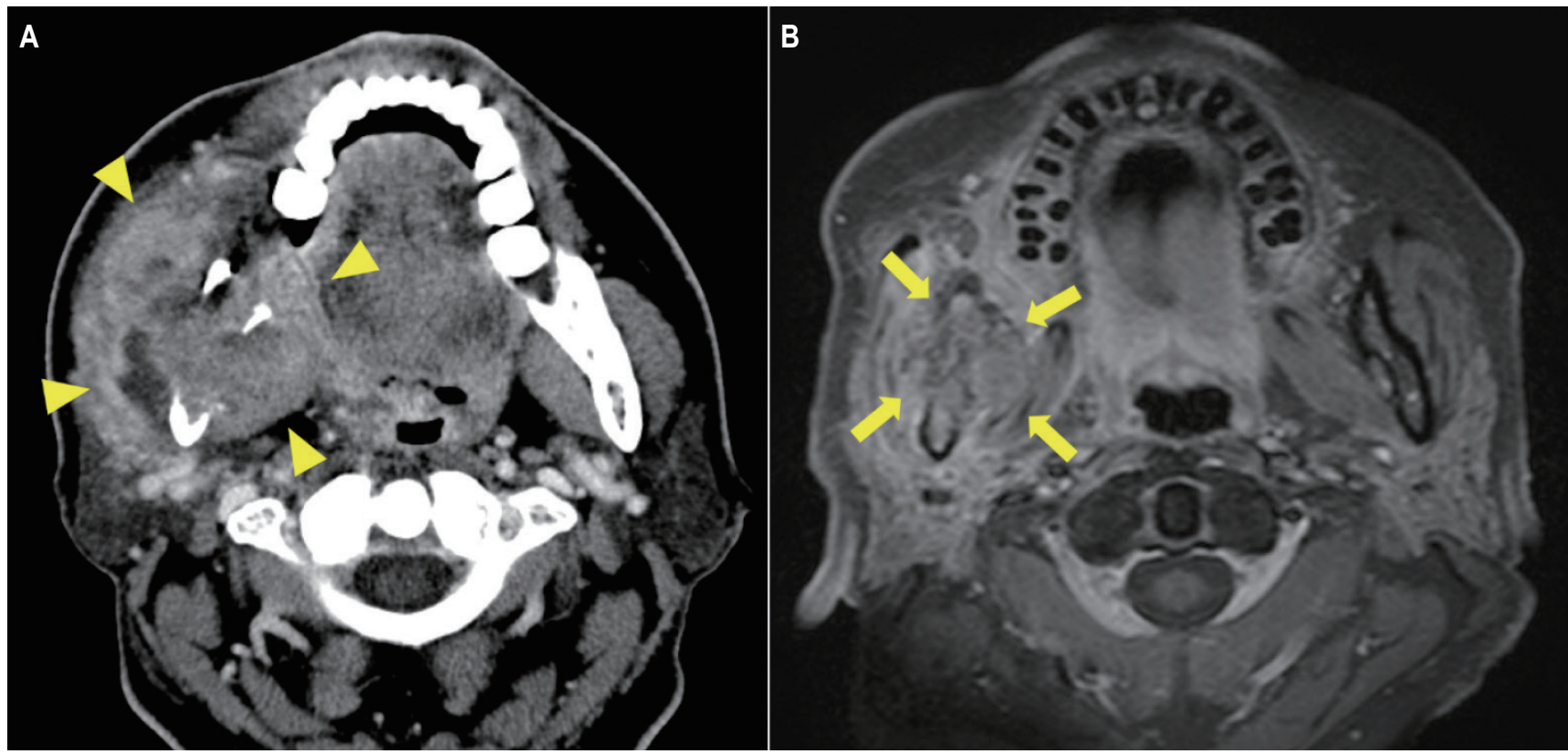

Fig. 1. (A) A huge soft tissue swelling with a heterogeneously enhancing mass $(7.2 \mathrm{~cm} \times 5.8 \mathrm{~cm})$ in the right masticator muscle and right side of mandibular ramus on computed tomography (yellow triangles) prior radiotherapy. (B) A decreased soft tissue mass (3.9 $\mathrm{cm}$ $\times 2.7 \mathrm{~cm}$ ) on magnetic resonance image (yellow arrows) 4 months after radiotherapy.

In our current analysis, only 1 patient had a lung metastasis whereas none of the remaining 8 cases was diagnosed with a pulmonary metastasis from HCC, suggesting that the Batson's venous plexus was the possible main route of dissemination to the mandible in these patients.

Based on previous reported cases, most patients diagnosed with a mandibular metastasis from HCC have been men (86.3\%) with a median age of 61 years (range, 16 to 88 years; Supplementary Table S2). A mandibular metastasis has also been the first detected manifestation of a malignancy before the diagnosis of the primary HCC in $67.2 \%$ of all currently available cases. It must be noted however that a mandibular metastasis was diagnosed during treatment and was accompanied by other extrahepatic metastases in most of the patients in this current study. The most common symptoms were swelling (72.1\%), followed by pain (32.4\%), paresthesia or numbness (29.4\%), bleeding (16.2\%), difficulty in mouth opening (7.4\%) and tooth loosening (2.9\%) in these patients (Supplementary Table S2). Almost half of the mandibular metastases in our present study series occurred in the ramus including the condyle. Previous reports have indicated that the body is a common site (35.4\%) of a mandibular metastasis but this was not found in our current cases. Our current patients more frequently suffered from pain (88.9\%) and difficulty in mouth opening (55.6\%), whilst swelling (33.3\%) was less common, compared with previous studies. This may have been due to the higher rate of condyle involvement in our current study cases. The mandibular condyle articulates with the glenoid fossa of the temporal bone in the temporomandibular joint and has an essential functional role as it acts as a hinge $[19,20]$. A condyle metastasis can cause dislocation of the joint or movement disruption, accompanied by severe pain.

Biopsies were used to confirm the diagnosis of a bone metastasis from HCC, particularly in cases with no history of malignancy. However, a severe hemorrhage after incisional or excisional biopsy has been reported to be relatively common in previous case reports $[7,8,10,13]$. Bone metastases from HCC commonly have an expansile mass that causes destruction of the involved bone, which is characterized by a similar hypervascularization to that of the primary HCC [4]. Furthermore, most HCC patients have a high risk of bleeding due to an underlying liver cirrhosis [21]. Special care should thus be taken when performing an incisional biopsy for a mandibular mass in patients with HCC. Moreover, a biopsy could be avoided in these cases when a typical finding of a bone metastasis from HCC is evident on a diagnostic CT or MRI.

Radiotherapy is a proven palliative treatment for bone metastases and has also been shown to provide pain relief in $70 \%-99.5 \%$ of bone metastases arising from HCC $[4,5,22]$ 

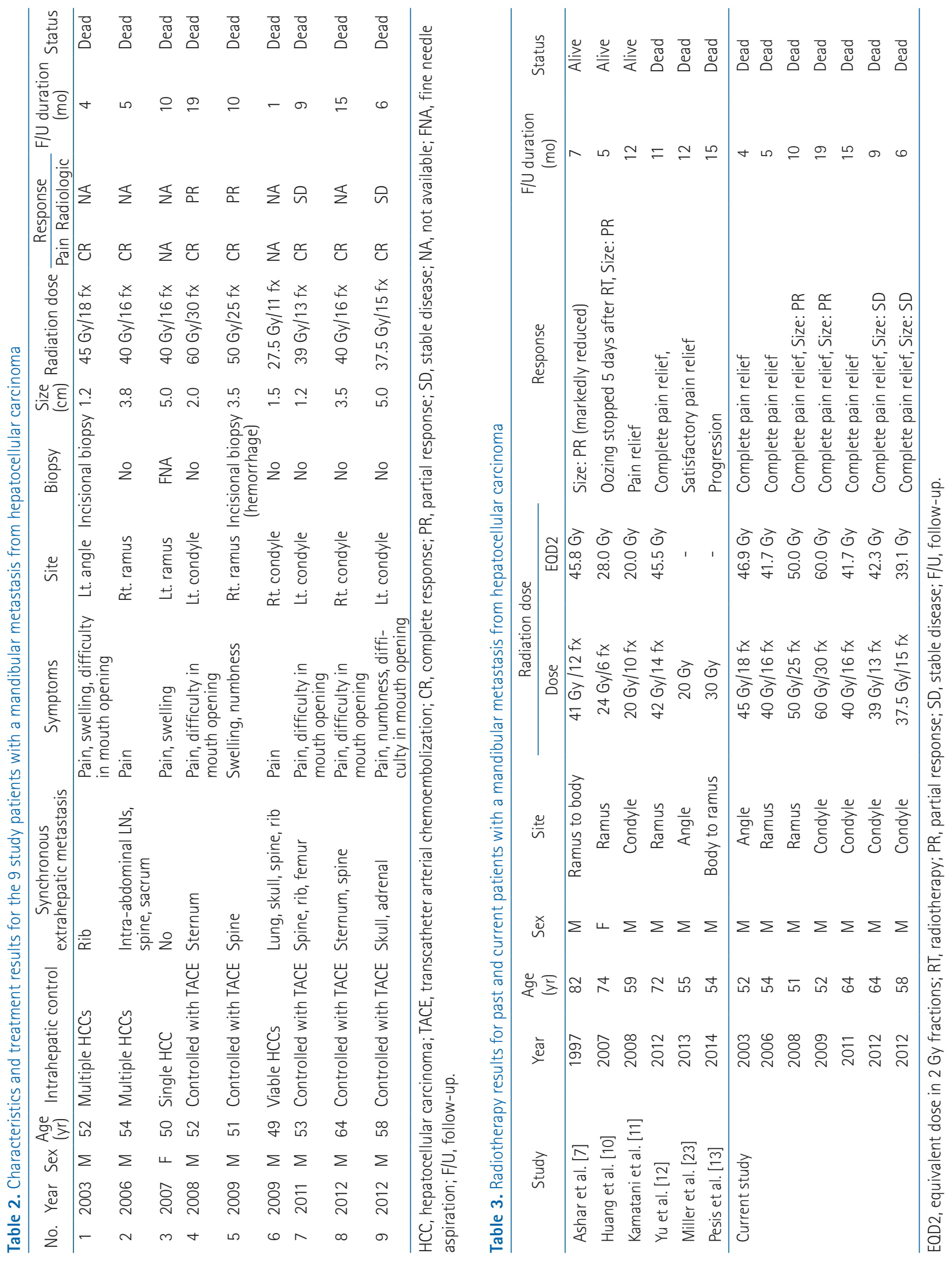
Detailed information on the outcomes of radiotherapy for a mandibular metastasis from HCC is scant in the current literature however. Among the 77 cases that have been reported to date, 45 patients received a local treatment for the mandibular metastasis (Supplementary Table S2). Radiotherapy was the most commonly used local treatment modality, including radiotherapy after surgery. Notably, the prescribed radiation dose and treatment response have been specified in only 6 cases $[7,10-13,23]$. Table 3 summarizes the radiotherapy results in these 6 reported cases and in the 7 patients in our current series. Although various radiation doses and fractionations have been used to treat mandibular lesions (median, $39 \mathrm{~Gy}$; range, 20 to $60 \mathrm{~Gy}$ ), symptoms are reported to have been successfully relieved by radiotherapy in most cases. The median prescribed EQD2 was $41.7 \mathrm{~Gy}$, which was higher than the conventional palliative radiotherapy dose of $30 \mathrm{~Gy}$ in 10 fractions (EQD2, 32.5 Gy). Therefore, it would be necessary to deliver higher than conventional radiation doses to control the symptoms related to mandibular metastasis. Although the prognosis of a mandibular metastasis from HCC is very poor according to our current findings, particularly in patients with uncontrolled intrahepatic HCCs, an active palliative radiotherapy approach should be considered to improve the $\mathrm{OoL}$ in these patients. In patients expected to have poor survival and with a poor performance status, a short-course of palliative radiotherapy should also be considered.

There were several limitations to this study of note. First, a small number of patients with heterogeneous clinical conditions were included, although it must be noted that only 77 cases of a mandibular metastasis from HCC have been described in the past 60 years. Our current cohort is thus one of the largest series to be used to assess treatment outcomes in patients with this condition. Moreover, few studies have described the results of radiotherapy as a palliative treatment for bone metastasis. Our current findings thus provide valuable information regarding this rare metastasis. Second, our data have a potential bias and should therefore be interpreted with caution because of the retrospective nature of our analyses and the incompleteness of the clinical data. It was also difficult to evaluate treatment-related toxicity after palliative radiotherapy. Third, various radiation doses were administered in our cohort in accordance with the patients' conditions making it difficult to suggest an optimal radiotherapy schedule for a mandibular metastasis from HCC.

In conclusion, mandibular metastases arising from HCCs are associated with an extremely poor prognosis. Because most affected patients show various symptoms including pain, radiotherapy should be considered for palliation in these cases, notwithstanding the low expected survival rates.

\section{Conflict of Interest}

No potential conflict of interest relevant to this article was reported.

\section{Supplementary Materials}

Supplementary materials can be found via http://doi. org/10.3857.roj.2019.00479. Table S1. Summary of the reported cases of a mandibular metastasis from hepatocellular carcinoma. Table S2. Characteristics of the reported cases with a mandibular metastasis from hepatocellular carcinoma.

\section{References}

1. Ferlay J, Soerjomataram I, Dikshit R, et al. Cancer incidence and mortality worldwide: sources, methods and major patterns in GLOBOCAN 2012. Int J Cancer 2015;136:E359-86.

2. Natsuizaka M, Omura T, Akaike T, et al. Clinical features of hepatocellular carcinoma with extrahepatic metastases. J Gastroenterol Hepatol 2005;20:1781-7.

3. Uchino K, Tateishi R, Shiina S, et al. Hepatocellular carcinoma with extrahepatic metastasis: clinical features and prognostic factors. Cancer 2011;117:4475-83.

4. He J, Zeng ZC, Tang ZY, et al. Clinical features and prognostic factors in patients with bone metastases from hepatocellular carcinoma receiving external beam radiotherapy. Cancer 2009;115:2710-20.

5. Seong J, Koom WS, Park HC. Radiotherapy for painful bone metastases from hepatocellular carcinoma. Liver Int $2005 ; 25: 261-5$.

6. Dick A, Mead SG, Mensh M, Schatten WE. Primary hepatoma with metastasis to the mandible. Am J Surg 1957;94:846-50.

7. Ashar A, Khateery SM, Kovacs A. Mandibular metastatic hepatocellular carcinoma: a case involving severe postbiopsy hemorrhage. J Oral Maxillofac Surg 1997;55:547-52.

8. Junquera L, Rodriguez-Recio C, Torre A, Sanchez-Mayoral $J$, Fresno MF. Hepatocellular carcinoma metastatic to the mandible: a case involving severe hemorrhage. Med Ora 2004;9:345-9.

9. Pires FR, Sagarra R, Correa ME, Pereira CM, Vargas PA, Lopes MA. Oral metastasis of a hepatocellular carcinoma. Oral Surg Oral Med Oral Pathol Oral Radiol Endod 2004;97:359-68.

10. Huang SF, Wu RC, Chang JT, et al. Intractable bleeding from 
solitary mandibular metastasis of hepatocellular carcinoma. World J Gastroenterol 2007;13:4526-8.

11. Kamatani T, Tatemoto $Y$, Tateishi Y, Yamamoto T. Isolated metastasis from hepatocellular carcinoma to the mandibular condyle with no evidence of any other metastases: a case report. Br J Oral Maxillofac Surg 2008;46:499-501.

12. Yu S, Estess A, Harris W, Dillon J. A rare occurrence of hepatocellular carcinoma metastasis to the mandible: report of a case and review of the literature. J Oral Maxillofac Surg 2012;70:1219-23.

13. Pesis M, Taicher S, Greenberg G, Hirshberg A. Metastasis to the jaws as a first manifestation of hepatocellular carcinoma: report of a case and analysis of 41 cases. J Craniomaxillofac Surg 2014;42:1997-2001.

14. Bruix J, Sherman M; American Association for the Study of Liver Diseases. Management of hepatocellular carcinoma: an update. Hepatology 2011;53:1020-2.

15. Chow E, Hoskin P, Mitera G, et al. Update of the international consensus on palliative radiotherapy endpoints for future clinical trials in bone metastases. Int J Radiat Oncol Biol Phys 2012;82:1730-7.

16. Servato JP, de Paulo LF, de Faria PR, Cardoso SV, Loyola AM.
Metastatic tumours to the head and neck: retrospective analysis from a Brazilian tertiary referral centre. Int J Oral Maxillofac Surg 2013;42:1391-6.

17. Hirshberg A, Shnaiderman-Shapiro A, Kaplan I, Berger R. Metastatic tumours to the oral cavity: pathogenesis and analysis of 673 cases. Oral Oncol 2008;44:743-52.

18. Tobinick E, Vega CP. The cerebrospinal venous system: anatomy, physiology, and clinical implications. MedGenMed 2006;8:53.

19. Lomas J, Gurgenci T, Jackson C, Campbell D. Temporomandibular dysfunction. Aust J Gen Pract 2018;47:212-5.

20. Liddell A, Perez DE. Temporomandibular joint dislocation. Oral Maxillofac Surg Clin North Am 2015;27:125-36.

21. Tripodi A, Mannucci PM. The coagulopathy of chronic liver disease. N Engl J Med 2011;365:147-56.

22. Jung IH, Yoon SM, Kwak J, et al. High-dose radiotherapy is associated with better local control of bone metastasis from hepatocellular carcinoma. Oncotarget 2017;8:15182-92.

23. Miller ME, McCall AA, Juillard GF, Nadelman CM, Wang MB, Nabili V. Hepatocellular carcinoma metastatic to the mandible. Ear Nose Throat J 2013;92:E17-9. 